\title{
Anatomical basis of approaches to foramen magnum and lower clival meningiomas: comparison of retrosigmoid and transcondylar approaches
}

\author{
Agustinus Suhardja, M.D., Anne M. R. Agur, B.Sc. (OT), M.Sc., And \\ Michael D. Cusimano, M.D., FRCS(C), M.H.P.E., PH.D., D.A.B.N.S., F.A.C.S. \\ Division of Neurosurgery, St. Michael's Hospital, and Division of Anatomy and Cell Biology, \\ Department of Surgery, University of Toronto, Ontario, Canada
}

\begin{abstract}
Object. Meningiomas of the lower clivus and foramen magnum are among the most challenging of all neurosurgical lesions. Debate continues regarding the most appropriate approach to this eloquent anatomical region. This anatomical study was undertaken to measure and compare the area of surgical exposures of the lower clivus achieved using the retrosigmoid and the extreme-lateral transcondylar (ELT) approaches.

Methods. Thirteen embalmed cadaveric heads were dissected bilaterally via the retrosigmoid approach on one side and the ELT approach on the other. The circumference of the area of exposure was delineated using beaded pins placed into the dura. After removal of the brain, the longest longitudinal and transverse axes of the pinned areas were measured and surface area calculated. The area of surgical exposure was also expressed as a percentage of the total area of the lower clivus. Normalized and adjusted surface areas were calculated using the bimastoid diameter. The areas of exposure were compared using the two-tailed paired Student t-test.

The mean area of exposure required using the retrosigmoid approach was $19.8 \pm 14.7 \mathrm{~mm}^{2}\left(\right.$ range $\left.6-49 \mathrm{~mm}^{2}\right)$ and that using the ELT approach was $27.8 \pm 22.8 \mathrm{~mm}^{2}$ (range $10-90 \mathrm{~mm}^{2}$ ). The mean percentage of the lower clivus exposed by the retrosigmoid approach was $14.9 \pm 3.6 \%$ (range 10-22\%) and that exposed by the ELT approach was $20.5 \pm 4.9 \%$ (range 10-25\%). The ELT approach provided significantly greater area of operative exposure and allowed a significantly higher percentage of lower clivus and foramen magnum exposure than did the retrosigmoid approach $(\mathrm{p}<0.05)$. Normalized and adjusted surface areas, taking into consideration the bimastoid diameter, were also statistically significant in favor of the ELT approach.

Conclusions. The ELT approach provided a significantly greater area of exposure than did the retrosigmoid approach.
\end{abstract}

\section{KEY WORDS • meningioma • clivus • foramen magnum • retrosigmoid approach • extreme-lateral transcondylar approach • skull base}

There has been a surge of interest in the development of skull base approaches in the last three decades..$^{1,5,6,10,12 \text {, }}$ 13,15,22 These approaches were devised with the aim of improving the exposure and removal of skull base lesions by performing extensive soft-tissue dissection and bone removal, thereby minimizing brain retraction. It has been argued, however, that skull base approaches increase invasiveness without offering the surgeon additional operative exposure. . $^{16,27}$

Debate over the extreme-lateral transoccipitocondylar approach exemplifies this controversy. The ELT approach (or, more simply stated, the transcondylar approach) was developed as an alternative to the retrosigmoid approach (also known as the suboccipital approach or suboccipital

Abbreviations used in this paper: $\mathrm{CCJ}=$ craniocervical junction; ELT $=$ extreme-lateral transcondylar; $\mathrm{SD}=$ standard deviation; $\mathrm{VA}=$ vertebral artery. retrosigmoid approach) for facilitating removal of lesions such as meningiomas located in the lower clivus and anterior foramen magnum. ${ }^{3,25}$ The basic premise is that the surgeon, attempting to reach the lower clivus, obtains wider soft-tissue exposure and more bone removed without risking neurovascular injury by retraction of critical neurovascular structures, such as the VA and medulla oblongata. This approach, however, is time consuming and may compromise the integrity of the CCJ because of the extensive drilling of the occipital condyle. ${ }^{2}$ Some authors ${ }^{2,20}$ have suggested that the approach is not necessary, whereas others ${ }^{3}$ have asserted that it is indispensable for the treatment of lesions in this location.

To make a valid comparison, the ELT approach should be performed on one side and the retrosigmoid approach on the other in the same patient. Because such a study is not possible in the operating room, a controlled anatomical study may offer some insight into the area of surgical exposure provided by each approach. To our knowl- 
edge, no other study has addressed this issue. Therefore, the purpose of this study was to measure and compare the area of surgical exposures of the lower clivus and foramen magnum provided by the retrosigmoid and the ELT approaches.

\section{MATERIALS AND METHODS}

Thirteen embalmed cadaveric heads (26 sides) were dissected using the retrosigmoid approach on one side and the ELT approach on the other; an operating microscope was used. An electric drill with cutting and diamond burrs as well as and macro- and microsurgical instruments were used as necessary.

\section{Operative Approaches}

Retrosigmoid. The technique for the retrosigmoid approach has been previously described. ${ }^{17}$ Briefly, a reverse U-shaped incision was made in the suboccipital area. Skin and subcutaneous tissues were reflected inferiorly as a single layer. A suboccipital craniectomy was then performed with its superior extent just below the transverse sinus and its lateral extent just medial to the sigmoid sinus. The dura was opened in a cruciate fashion, and the occipital lobe was maximally retracted.

Extreme-Lateral Transcondylar. The technique for the ELT approach has been described by Sen, et al. ${ }^{24}$ The incision is extended along the upper third of the anterior border of the sternocleidomastoid muscle, to just above the lateral part of the superior nuchal line, to the midline, and then down the midline to C-4. After elevation of the skin flap along with the subcutaneous tissue, the posterior $\mathrm{C}-1$ arch and posterior portion of the C-1 lateral mass were removed using a cutting burr on a high-speed drill. ${ }^{4}$ The VA as well as its encircling venous plexus was then freed and mobilized from the C-1 transverse process. A standard suboccipital craniectomy ${ }^{17}$ was then performed. A partial mastoidectomy up to the vertical part of the facial nerve was conducted, and the sigmoid sinus was unroofed up to the level of the jugular bulb. The inferior extension of the craniectomy includes the foramen magnum and, laterally, the craniectomy includes drilling of the posterior half of the occipital condyle. The dural incision was made longitudinally, the dural edges tented up with sutures, and the occipital lobe retracted.

\section{Delineation of the Area of Exposure}

The area of surgical exposure was delineated by placing beaded pins into the dura around the circumference of the exposed area (Fig. 1). After removal of the brain, the longest longitudinal and transverse axes of the pinned areas were measured in millimeters. The area of exposure was then determined by multiplying the length of the two axes. The area of surgical exposure was expressed as a percentage of the total area of the lower clivus. The boundaries of the lower clivus were delineated, as detailed by Sekhar, et al., ${ }^{23}$ as follows: the upper border is a line joining the two jugular foramina; the lower border is a line running through the anterior margin of the foramen magnum; and each lateral border is formed by a line joining the jugular foramen and the hypoglossal canal.

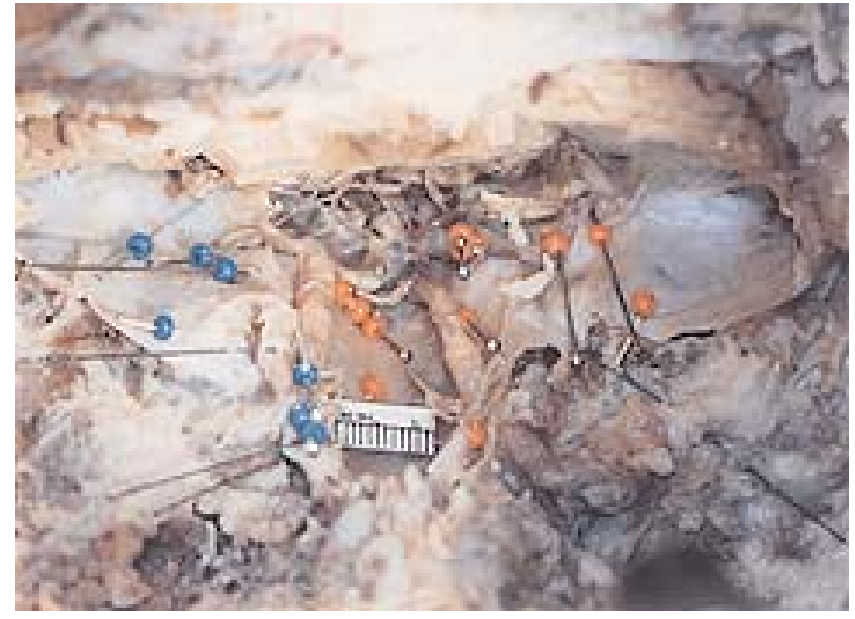

Fig. 1. Photograph of the clivus, posterior fossa, and foramen magnum. The area outlined by the orange pinheads demarcates the exposure provided by the ELT approach. The area demarcated by the blue pinheads on the left was provided by the retrosigmoid approach. The brainstem and inferior cerebellum have been resected. The cerebellar folia can be seen at the upper right corner. The ruler marks the location of the foramen magnum and is placed between the VAs.

\section{Statistical Analysis}

Data are expressed as mean \pm SD and assessed using the two-tailed Student paired t-test $(\mathrm{p}<0.05)$. Association between bimastoid diameter and area of surgical exposure was determined by use of the Pearson correlation coefficient. A normalized and adjusted surface area was calculated by multiplying and dividing, respectively, the surface area of exposure by the bimastoid diameter. The normalized and adjusted surface areas were assessed using the two-tailed Student paired t-test $(\mathrm{p}<0.05)$.

\section{RESULTS}

\section{Comparison of the Surface Area}

A summary of surface areas of exposure provided by the retrosigmoid and the ELT approaches is provided in Table 1 and Fig. 2 upper. The mean surface area of exposure provided by the retrosigmoid approach was $19.8 \pm$ $14.7 \mathrm{~mm}^{2}$ (range $6-49 \mathrm{~mm}^{2}$ ), and that provided by the ELT approach was $27.8 \pm 22.8 \mathrm{~mm}^{2}$ (range $10-90 \mathrm{~mm}^{2}$ ). The surface area of exposure provided by the ELT approach was significantly greater than that provided by the retrosigmoid approach $(p=0.024)$. The ELT approach provided a greater area of exposure of the lower clivus in 12 of 13 cadaveric heads examined. The areas of exposure created by both the retrosigmoid and ELT approaches were highly correlated with bimastoid diameter $(\mathrm{r}=0.86$ and 0.88 , respectively). Normalized surface area $(\mathrm{p}=$ $0.036)$ and adjusted surface area $(\mathrm{p}=0.016)$ were also significantly greater for the extreme-lateral approach.

\section{Percentage of the Lower Clivus Exposed}

The percentage of the lower clivus exposed by the retrosigmoid and the ELT approaches are summarized in 
Retrosigmoid and extreme-lateral approaches

TABLE 1

Summary of measured exposures achieved using the retrosigmoid and ELT approaches

\begin{tabular}{|c|c|c|c|}
\hline \multirow[b]{2}{*}{ Area Exposed } & \multicolumn{2}{|c|}{ Approach (range) } & \multirow{2}{*}{$\begin{array}{c}\mathrm{p} \\
\text { Value* }\end{array}$} \\
\hline & Retrosigmoid & ELT & \\
\hline total surface area & $\begin{array}{c}19.8 \pm 14.7 \mathrm{~mm}^{2} \\
\left(6-49 \mathrm{~mm}^{2}\right)\end{array}$ & $\begin{array}{c}27.8 \pm 22.8 \mathrm{~mm}^{2} \\
\left(10-90 \mathrm{~mm}^{2}\right)\end{array}$ & 0.024 \\
\hline $\begin{array}{l}\text { normalized surface } \\
\text { area } \dagger\end{array}$ & $\begin{array}{c}2241 \pm 1878 \mathrm{~mm}^{2} \\
\left(540-5978 \mathrm{~mm}^{2}\right)\end{array}$ & $\begin{array}{l}3144 \pm 2895 \mathrm{~mm}^{2} \\
\quad\left(1030-11070 \mathrm{~mm}^{2}\right)\end{array}$ & 0.036 \\
\hline $\begin{array}{l}\text { adjusted surface } \\
\text { area } \neq\end{array}$ & $\begin{array}{c}0.18 \pm 0.11 \mathrm{~mm}^{2} \\
\quad\left(0.07-0.40 \mathrm{~mm}^{2}\right)\end{array}$ & $\begin{array}{c}0.25 \pm 0.18 \mathrm{~mm}^{2} \\
\left(0.10-0.73 \mathrm{~mm}^{2}\right)\end{array}$ & 0.016 \\
\hline $\begin{array}{l}\% \text { total surface area } \\
\text { of clivus }\end{array}$ & $\begin{array}{c}14.9 \pm 3.6 \% \\
(10-22 \%)\end{array}$ & $\begin{array}{c}20.5 \pm 4.9 \% \\
(10-25 \%)\end{array}$ & 0.002 \\
\hline
\end{tabular}

* Determined by performing the two-tailed paired Student t-test.

$\dagger$ The bimastoid diameter multiplied by the total surface area of exposure.

\$ The total surface area of exposure divided by the bimastoid diameter.

Table 1 and Fig. 2 lower. The mean percentage of the lower clivus exposed via the retrosigmoid approach was $14.9 \pm 3.6 \%$ (range 10-22\%) and that exposed by the ELT approach was $20.5 \pm 4.9 \%$ (range 10-25\%). The percentage of the lower clivus exposed by the ELT approach was significantly greater than that by the retrosigmoid approach $(\mathrm{p}=0.002)$.

\section{DISCUSSION}

Notoriously known for their association with a high surgery-related morbidity rate, ventrally located lesions in the CCJ have inspired various alternatives to the traditional retrosigmoid approach. The ELT approach has become increasingly more extensive in its area of exposure. In addition to the large occipital craniotomy, ${ }^{11}$ Sen and Sekhar $^{26}$ drilled half of the occipital condyle ${ }^{9}$ and later Sen and Sekar, ${ }^{26}$ and Babu, et al., ${ }^{3}$ drilled the entire occipital condyle. George, et al., ${ }^{7}$ and others ${ }^{24}$ recommended partial mastoidectomy, Bertalanffy and Seeger ${ }^{4}$ recommended drilling of the atlantal lateral mass, and ultimately George, et al. ${ }^{8}$ and Sen and Sekar ${ }^{26}$ added sectioning of the sigmoid sinus. The approach has gained wide acceptance because it avoids entering the contaminated oral cavity, provides early visualization of the VA, and creates wider surgical exposure. ${ }^{4,8}$ Although there is little argument regarding these advantages, the ELT approach has become a subject of debate between proponents and opponents of the approach. Patterson ${ }^{19}$ and Samii, et al., ${ }^{20}$ have argued that most lesions of the lower clivus can be removed without the time-consuming extensive dissection of the ELT approach. Furthermore, they consider condylar resection unnecessary, potentially contributing significantly to the morbidity rate by requiring occipitocervical fusion.

In this study we found the mean difference between the surface area of exposure provided by the ELT and the retrosigmoid approaches to be $8 \mathrm{~mm}^{2}(\mathrm{p}<0.05)$. The additional drilling of the occipital condyle and partial mastoidectomy provided by the ELT approach expanded the area of exposure of the retrosigmoid approach anteriorly and ventrally. The exposure provided by the ELT approach, when $50 \%$ of the occipital condyle is drilled, however, is limited to the midline. Further extension of the exposure across the midline may be achieved by drilling

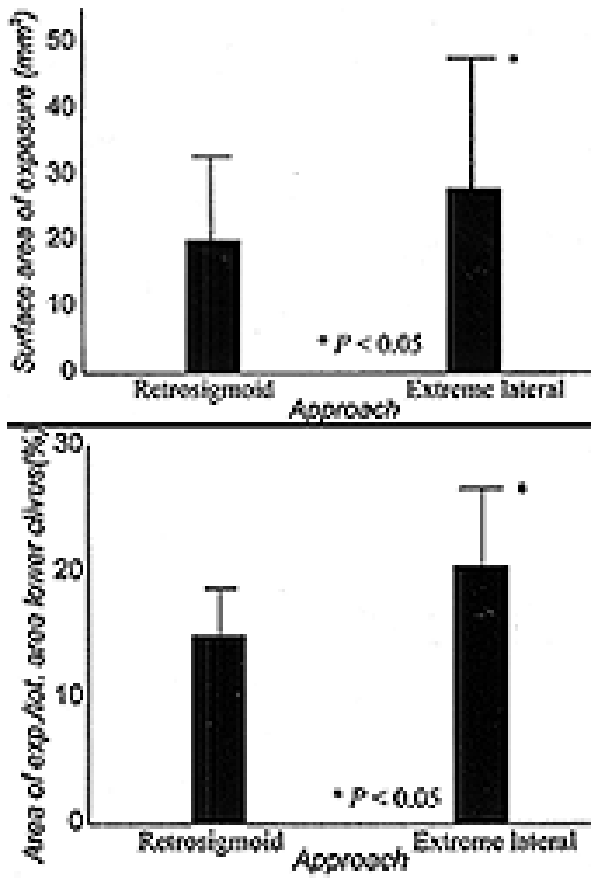

Fig. 2. Upper: Bar graph demonstrating the mean surface area of exposure provided by the two approaches. Values indicated by the bars and vertical lines are the mean $\pm \mathrm{SD}$, respectively, of the area of exposure provided by each approach. The asterisk denotes significantly greater surface area of exposure provided by the ELT approach $(\mathrm{p}<0.05)$. Lower: Bar graph demonstrating the percentage of the area of the lower clivus exposed by the two approaches. Values represent mean percentage $\pm S D$, respectively, of the exposure area created by each approach. The asterisk denotes the significantly greater percentage area of exposure achieved using the ELT approach $(\mathrm{p}<0.05)$.

the entire occipital condyle. ${ }^{3}$ The benefits of such wide exposure, however, have to be weighed against the risk of compromising the stability of the CCJ that may result from resecting of the entire condyle.

In our specimens, the retrosigmoid approach exposed $14.9 \%$ of the lower clivus and anterior foramen magnum, whereas $20.5 \%$ of the region was exposed via the ELT approach. These values are consistent with other studies in which the authors have indicated that additional structures such as vertebrobasilar junction, and proximal posterior inferior cerebellar artery, and sometimes the contralateral VA can be exposed via the ELT approach. ${ }^{14,18,21}$

The results of this study demonstrate a wide range and SD in values, which is explained by variability of head size (that is, the bimastoid diameter). The bimastoid diameter was found to be highly correlated with the area of exposure. Therefore, the normalized and adjusted surface areas, in which differences in head size are taken into account, further confirm the statistically significant difference of the exposure area provided by the ELT and the retrosigmoid approaches.

We found that it may be appropriate to access lesions on the lower clivus and anterior foramen magnum that require wider exposure gained through the ELT approach. Larger slow-growing lesions not extending beyond the midline, however, may already provide the needed "phys- 
iological retraction" and the creation of what the senior author (M.D.C.) calls a three-dimensional surgical corridor that not only provides access but also allows the instrument-related maneuverability. The surgical corridor thus created by larger lesions effectively allows an extreme-lateral exposure without the need to drill the condyle (Fig. 3). More extensive lesions, such as those that cross the midline, may require even wider exposure by removing the entire condyle. Although drilling of the jugular tubercle can also improve visualization of the lower clivus, this was not the object of our study. Clearly it is this concept of a surgical corridor that can explain differences in surgery-related philosophy concerning this region. ${ }^{8,20}$ In our experience, the surgical corridor is assessed on preoperative imaging, and the retrosigmoid approach is used in cases involving larger and more laterally located lesions that create a larger corridor and the transcondylar approach is used in cases involving smaller more ventrally situated lesions. Ultimately clinical studies cannot resolve the issue of surgical approach alone because lesions in this region are rare and randomization is unlikely. Clearly, the astute surgeon will individualize the approach to the specific anatomy of each lesion and patient.

\section{CONCLUSIONS}

The ELT approach provided a significantly greater area of exposure of the lower clivus and foramen magnum than the retrosigmoid approach.

\section{Acknowledgments}

We thank John Reddy and Scott Riddell of Leica Microsystems

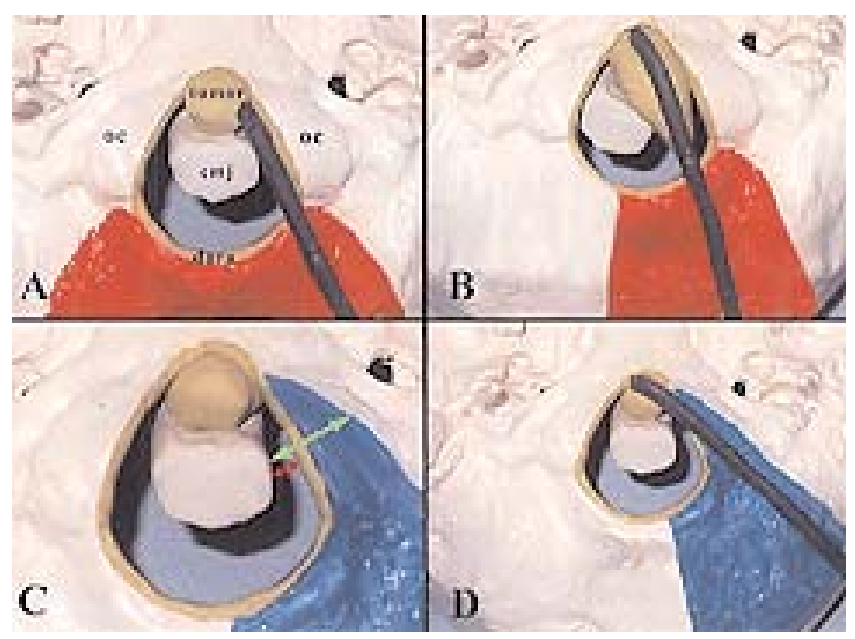

Fig. 3. Photographs showing the surgical corridor to anterior foramen magnum and lower clival meningiomas. A: Suboccipital craniotomy (red) with a narrow corridor does not allow effective exposure of the tumor for resection. $\mathrm{CMJ}=$ cervicomedullary junction. B: Tumor growth naturally widens the surgical corridor, allowing safe and effective removal via suboccipital craniotomy without drilling of the condyle. C and D: Transcondylar exposure (blue) widens the corridor by removing the medial condyle (red arrow represents very narrow corridor before condyle resection, green arrow represents adequate corridor after its removal) (C), thereby allowing access to much of the tumor (D).
(Canada), Inc., Thomas Klingebiel of Carl Zeiss Canada, Ltd., and Todd McDowell of Codman Surgical Company for their technical support. We are also grateful to Jackie Grummitt who provided manuscript assistance, Jianli Li, Ph.D., for his advice and helpful suggestions in the statistical analysis, and Alex McDonald for assistance with the figures.

\section{References}

1. Al-Mefty O, Fox JL, Smith RR: Petrosal approach for petroclival meningiomas. Neurosurgery 22:510-517, 1988

2. Arnold H, Sepehrnia A: Extreme lateral transcondylar approach. J Neurosurg 82:313-314, 1995 (letter)

3. Babu RP, Sekhar LN, Wright DC: Extreme lateral transcondylar approach: technical improvements and lessons learned. J Neurosurg 81:49-59, 1994

4. Bertalanffy H, Seeger W: The dorsolateral, subocciptal, transcondylar approach to the lower clivus and anterior portion of the craniocervical junction. Neurosurgery 29:815-821, 1991

5. Crockard HA: The transoral approach to the base of the brain and the upper cervical cord. Ann R Coll Surg Engl 67: 321-325, 1985

6. Fisch U: Infratemporal fossa approach for glomus tumors of the temporal bone. Ann Otol Rhinol Laryngol 91:474-479, 1982

7. George B, Dematons C, Cophignon J: Lateral approach to the anterior portion of the foramen magnum. Application to surgical removal of 14 benign tumors: technical note. Surg Neurol 29:484-490, 1988

8. George B, Lot G, Boissonnet H: Meningioma of the foramen magnum: a series of 40 cases. Surg Neurol 47:371-379, 1997

9. Gilsbach JM, Eggert HR, Seeger W: The dorsolateral approach in ventrolateral craniospinal lesions, in Voth D, Glees P (eds): Diseases in the Cranio-Cervical Junction: Anatomical and Pathological Aspects and Detailed Clinical Accounts. Berlin: Walter de Gruyter, 1987, pp 359-364

10. Hakuba A, Liu SS, Nishimura S: The orbitozygomatic infratemporal approach: a new surgical technique. Surg Neurol 26: 271-276, 1986

11. Heros RC: Lateral suboccipital approach for vertebral and vertebrobasilar artery lesions. J Neurosurg 64:559-562, 1986

12. House WF, Hitselberger WE: The transcochlear approach to the skull base. Arch Otolaryngol 102:334-342, 1976

13. Janecka IP, Sen CN, Sekhar LN, et al: Facial translocation: a new approach to the cranial base. Otolaryngol Head Neck Surg 103:413-419, 1990

14. Kawase T, Bertalanffy H, Otani M, et al: Surgical approaches for vertebro-basilar trunk aneurysms located in the midline. Acta Neurochir 138:402-410, 1996

15. Kawase T, Toya S, Shiobara R, et al: Transpetrosal approach for aneurysms of the lower basilar artery. J Neurosurg 63: $857-861,1985$

16. Ojemann RG: Skull-base surgery: a perspective. J Neurosurg 76:569-570, 1992

17. Ojemann RG: Suboccipital transmeatal approach to vestibular schwannomas, in Schmidek HH, Sweet WH (eds): Operative Neurosurgical Techniques, ed 3: Philadelphia: WB Saunders, 1995, Vol. 1, pp 829-842

18. Origitano TC, Anderson DE, Tarassoli Y, et al: Skull base approaches to complex cerebral aneurysms. Surg Neurol 40: 339-346, 1993

19. Patterson RH: Comment on George B, Lot G, Boissonet H: Meningioma of the foramen magnum: a series of 40 cases. Surg Neurol 47:371-379, 1997

20. Samii M, Klekamp J, Carvalho G: Surgical results for meningiomas of the craniocervical junction. Neurosurgery 39: 1086-1095, 1996

21. Sekhar LN, Kalia KK, Yonas H, et al: Cranial base approaches to intracranial aneurysms in the subarachnoid space. Neurosurgery 35:472-483, 1994 


\section{Retrosigmoid and extreme-lateral approaches}

22. Sekhar LN, Schramm VL Jr, Jones NF: Subtemporal-preauricular infratemporal fossa approach to large lateral and posterior cranial base neoplasms. J Neurosurg 67:488-499, 1987

23. Sekhar LN, Sen C, Snyderman CH, et al: Anterior, anterolater$\mathrm{al}$, and lateral approaches to extradural petroclival tumors, in Sekhar LN, Janecka IP (eds): Surgery of Cranial Base Tumors. New York: Raven Press, 1993, pp 157-223

24. Sen CN, Sekhar LN: An extreme lateral approach to intradural lesions of the cervical spine and foramen magnum. Neurosurgery 27:197-204, 1990

25. Sen CN, Sekhar LN: Extreme lateral approach to intradural lesions of the cervical spine and foramen magnum. Neurosurgery 28:779, 1991 (letter)
26. Sen CN, Sekhar LN: Surgical management of anteriorly placed lesions at the craniocervical junction-an alternative approach. Acta Neurochir 108:70-77, 1991

27. Tatagiba M, Samii M, Matthies C, et al: Management of petroclival meningiomas: a critical analysis of surgical treatment. Acta Neurochir Suppl 65:92-94, 1996

Manuscript received April 16, 2003.

Accepted in final form May 8, 2003.

Address reprint requests to: Michael D. Cusimano, M.D., Ph.D., Saint Michael's Hospital, University of Toronto, 2004-38 Shuter Street, Toronto, Ontario, Canada M5B 1A6. email: mountain@ smh.toronto.on.ca. 